\title{
PREVALÊNCIA DA DOR LOMBAR EM ENFERMEIROS EM CONTEXTO HOSPITALAR
}

\author{
Pedro Miguel Santos ${ }^{1}$ \\ Rosa Martins ${ }^{2}$ \\ Florentino Serranheira ${ }^{3}$
}

\begin{abstract}
Resumo: Enquadramento: A dor lombar é uma ocorrência bastante frequente, sobretudo em enfermeiros que trabalham em contexto hospitalar. As suas determinantes são variadas, podendo ser de cariz individual, psicossocial e profissional/organizacional.

Objetivo: Identificar a prevalência $e$ as determinantes da lombalgia em enfermeiros que trabalham em contexto hospitalar. Pretende-se ainda procurar relações entre os sintomas auto-referidos e a avaliação clínica de sinais, contribuindo para a prevenção.

Método: Trata-se de um estudo transversal, descritivocorrelacional e quantitativo. Numa primeira fase foi aplicado um questionário sobre lombalgia a 135 enfermeiros de um hospital em Lisboa. Posteriormente foi realizada uma avaliação clínica a 48 desses enfermeiros.

Resultados: Os resultados evidenciam uma elevada prevalência de lombalgia (60.7\%) nos enfermeiros da amostra, estando esta associada a algumas variáveis sociodemográficas, organizacionais e profissionais. Destacam-se: o grupo etário $(p=0.016)$, a altura $(p=0.035)$, o trabalho por turnos $(p=0.044)$ e o tempo de profissão $(p=0.005)$. De forma análoga, o "posicionamento/mobilização do doente na cama" $(p=0.026)$, o "levantar o doente da cama sem ajuda mecânica" ( $p=0.004)$, o "trabalho de pé" (65.9\%), o "inclinar (86.6\%) e rodar (76.8\%) o tronco" e a "manipulação de cargas" $(85.4 \%)$ revelaram-se influentes na dor lombar.

Relativamente à avaliação clínica, dos enfermeiros avaliados $(n=48), 89.6 \%$ referiram dor lombar com predominância de
\end{abstract}

\footnotetext{
${ }^{1}$ Enfermeiro, Centro Hospitalar Tondela-Viseu, Av. Rei D. Duarte, 3504-509 Viseu, Portugal.Email: pmsantos9@gmail.com

2 Professora Coordenadora da Escola Superior de Saúde do Instituto Politécnico de Viseu. Email: rmmartins.viseu@gmail.com

3 Ergonomista, Escola Nacional de Saúde Pública, Universidade Nova de Lisboa, Avenida Padre Cruz, 1600-560 Lisboa, Portugal

CISP - Centro de Investigação em Saúde Pública; CMDT - Centro de Investigação em Malária e Doenças Tropicais
} 
intensidade moderada $(n=38)$, sendo que os sintomas se mantiveram desde a resposta ao questionário.

Conclusões: Conclui-se, neste estudo, que a dor lombar assume especial relevância na profissão de enfermagem. A lombalgia dos enfermeiros que trabalham em meio hospitalar é não específica e apresenta caraterísticas mecânicas. Os fatores de risco relacionados com a atividade, presentes nas tarefas desempenhadas, são os que mais contribuem para a existência de lombalgia. Recomendam-se intervenções que se baseiem na avaliação dos fatores de prognóstico de cronicidade, de modo a evitar a evolução desfavorável da lombalgia e prevenir o aparecimento de novos casos.

Palavras-chave: dor lombar; enfermeiros; hospital; fatores de risco; caraterísticas do trabalho; prevenção.

\title{
Title: LOW BACK PAIN PREVALENCE IN NURSES IN HOSPITAL CONTEXT
}

\begin{abstract}
Background: Low back pain is common in nurses working in hospitals. The causes for this pain are various and can have its origins in individual, psychosocial and professional/organizational factors.

Objective: To identify the prevalence and the causes of low back pain in nurses working in hospitals and relate the self-reported symptoms with clinical evaluation in order to contribute to the prevention.

Method: This study is cross-sectional, descriptive-correlative and quantitative. Initially a questionnaire was applied on low back pain (adaptation of the Nordic Musculoskeletal Questionnaire) to 135 nurses at a hospital in Lisbon. Later was performed a clinical evaluation of 48 of these nurses.

Results: The results show a high prevalence of low back pain $(60.7 \%)$ in the sample nurses, this being associated with some sociodemographic, organizational and professional variables. Stand out from the age group $(p=0.016)$, height $(p=0.035)$, shift work $(p=0.044)$ and length of service $(p=0.005)$. Also "the positioning/mobilization of patient in bed" $(p=0.026)$, "get the patient out of bed without mechanical assistance" ( $p=0.004)$, "standing" (65.9\%), "tilting" (86.6\%) and rotating (76.8\%) the trunk" and "handling charges" (85.4\%) proved related to the presence of low back pain.

Concerning to clinical evaluation, $89.6 \%$ of nurses involved $(n=48)$ reported suffering from low back pain with moderate intensity $(n=38)$, and the symptoms remained since the response to the questionnaire.

Conclusions: With this study, we come to the conclusion that low back pain is particularly relevant in the nursing profession. Low back pain of nurses working in hospitals is non-specific and has
\end{abstract}


mechanical characteristics. Risk factors related to the activity, present in the tasks performed, are the most important to the existence of low back pain. It is recommended to work on interventions that are based on the evaluation of prognostic factors for chronicity in order to avoid the unfavorable evolution of low back pain and to prevent the appearance of new cases.

Keywords: Low back pain; nurses, hospital, risk factors, work characteristics, prevention.

\section{INTRODUÇÃO}

A dor lombar (regularmente designada por lombalgia) é um problema de saúde muito frequente e afeta a maioria das pessoas em algum momento das suas vidas (Hoy, Brooks, Blyth, \& Buchbinder, 2010). Trata-se de alterações músculo-esqueléticas frequentemente relacionadas com o trabalho, dando origem a doença profissional, sendo a maior causa de morbilidade em todo o mundo (Hoy, et al., 2010). A dor lombar tem um enorme impacto não só a nível individual, mas também familiar e social constituindo um elevado fardo económico no domínio da saúde (Dagenais, Caro, \& Haldeman, 2008).

Os enfermeiros são os profissionais mais afetados pelas lesões músculo-esqueléticas ligadas ao trabalho (LMELT) em todo o mundo e é na sintomatologia localizada na região lombar que o problema assume proporções mais preocupantes (Serranheira, Cotrim, Rodrigues, Nunes, \& Sousa-Uva, 2012; Tinubu, Mbada, Oyeyemi, \& Fabunmi, 2010).

Para uma avaliação mais rigorosa desta evidência apresentamos o quadro 1 que reflete a dimensão dos números já apurados, tanto a nível nacional como internacional.

De facto a profissão de enfermagem apresenta no seu dia a dia profissional uma componente física bastante exigente, uma vez que realizam frequentemente atividades que implicam posturas corporais extremas da coluna vertebral, particularmente da zona lombossagrada, tais como as transferências e os diferentes posicionamentos de doentes. Assim, a exposição a fatores de risco profissionais, como elevadas solicitações biomecânicas e fisiológicas que excedem as capacidades funcionais dos trabalhadores, aliados ao tipo de organização do trabalho, que não permite tempos de recuperação e repouso suficientes e adequados, conferem à profissão de enfermagem um risco acrescido de dor lombar para os seus profissionais (Cail, Aptel \& Franchi, 2000 citados por Serranheira, Cotrim, et al., 2012). 


\section{Quadro 1}

Prevalência de dor lombar nos enfermeiros: comparação de resultados em diferentes estudos

\begin{tabular}{|l|l|c|}
\hline \multicolumn{1}{|c|}{ País } & \multicolumn{1}{|c|}{ Estudo } & Dor lombar (\%) \\
\hline \multirow{2}{*}{ EUA } & $\begin{array}{l}\text { (Daraiseh, Cronin, Davis, Shell, \& Karwowski, } \\
\text { 2010) }\end{array}$ & 74.1 \\
\hline \multirow{2}{*}{ África } & (Sikiru \& Shmaila, 2009) & 70.9 \\
\cline { 2 - 3 } & (Tinubu, et al., 2010) & 44.1 \\
\hline \multirow{2}{*}{ Holanda } & (Alexopoulos, Burdorf, \& Kalokerinou, 2006) & 62.0 \\
\cline { 2 - 3 } & (Bos, Krol, van der Star, \& Groothoff, 2007) & 76.0 \\
\hline \multirow{2}{*}{ Grécia } & $\begin{array}{l}\text { (Alexopoulos, Burdorf, \& Kalokerinou, 2003; } \\
\text { Alexopoulos, et al., 2006) }\end{array}$ & 75.0 \\
\hline Israel & (Ratzon \& Froom, 2006) & 71.0 \\
\hline Inglaterra & (Smedley, Egger, Cooper, \& Coggon, 1995) & 60.0 \\
\hline Suíça & (Maul, Laubli, Klipstein, \& Krueger, 2003) & 73.0 a 76.0 \\
\hline Itália & (Lorusso, Bruno, \& L'Abbate, 2007) & 33.0 a 86.0 \\
\hline Reino Unido & (Branney \& Newell, 2009) & 54.1 \\
\hline Brasil & (Magnago et al., 2010) & 71.5 \\
\hline Turquia & (Karahan \& Bayraktar, 2004) & 85.7 \\
\hline \multirow{2}{*}{ Portugal } & (Fonseca \& Serranheira, 2006) $^{[}$ & 65.0 \\
\cline { 2 - 3 } & (Serranheira, Sousa-Uva, \& Sousa-Uva, 2012) & 60.9 \\
\hline
\end{tabular}

Um pouco por todo o mundo, estudos foram sendo feitos que documentam a problemática da dor lombar nos enfermeiros, com taxas de prevalência e incidência frequentemente elevadas (Daraiseh, et al., 2010; Magnago, et al., 2010; Sikiru \& Shmaila, 2009; Tinubu, et al., 2010), associadas a taxas de absentismo, aposentação precoce e diminuição da produtividade.

Recentemente, alguns estudos portugueses mostraram uma prevalência elevada de lombalgia entre os enfermeiros que desenvolvem a sua atividade profissional em ambiente hospitalar (Fonseca \& Serranheira, 2006; Serranheira, Sousa-Uva, et al., 2012), contudo, são escassos os estudos que refletem exclusivamente a realidade dos enfermeiros em meio hospitalar ou os que se ocupam unicamente desta patologia.

Com base nos pressupostos descritos, o presente estudo visa identificar a prevalência e as determinantes da dor lombar em enfermeiros que trabalham em ambiente hospitalar. 


\section{POPULAÇÃO E MÉTODOS}

Dos cerca de 400 enfermeiros do Hospital de Curry Cabral em Lisboa (população), 135 (amostra não probabilística por conveniência) aceitaram responder a um questionário online sobre sintomas músculo-esqueléticos ligados ao trabalho nos enfermeiros $(\mathrm{n}=135)$. Posteriormente, 48 desses enfermeiros concordaram ser submetidos a uma avaliação clínica por parte de dois fisiatras.

$\mathrm{O}$ instrumento de colheita de dados utilizado na primeira fase do estudo foi uma adaptação do questionário nórdico músculo-esquelético (QNM) que tem sido largamente utilizada em Portugal após ter sido devidamente validado, mas na sua forma reduzida, no sentido de restringir a colheita de dados à sintomatologia referida à região lombar (Serranheira, Cotrim, et al., 2012). Apesar disso, esta não deixa de contemplar as quatro dimensões do questionário original: (i) caraterização sociodemográfica; (ii) sintomatologia músculo-esquelética (dor lombar); (iii) tarefas de enfermagem desenvolvidas no trabalho e sua relação com os sintomas; (iv) caraterização do estado de saúde.

$\mathrm{Na}$ segunda fase do estudo (avaliação clínica) foi utilizada uma ficha de avaliação clínica com o intuito de uniformizar e objetivar a mesma. Elaborou-se um instrumento fácil e rápido de utilizar em consulta médica, mas eficaz no despiste de sinais de gravidade associados à presença de lombalgia, que consistia numa breve colheita de informação clínica referente a esta sintomatologia (início, frequência, duração, intensidade, qualidade, fatores atenuantes e exacerbantes, existência de radiculopatia irradiada ao membro) e num exame objetivo (Testes de Valsalva, Laségue e Romberg) $)^{4}$.

Foi desenvolvido um estudo quantitativo, não experimental, transversal e descritivo-correlacional. A análise estatística baseou-se em estatística descritiva e em associações através do teste Qui-quadrado, teste t-Student e teste de Fisher com nível de significância de 5\%.

\footnotetext{
${ }^{4}$ Teste de Valsalva: consiste em suster a respiração após inspiração profunda. $\mathrm{Na}$ compressão radicular a dor aumenta com esta manobra.

Teste de Laségue: consiste em elevar o membro inferior com o doente deitado em dorsal. É geralmente considerada positiva quando a dor irradia, ou se exacerba, no trajeto do dermátomo de L4-L5, ou L5-S1, quando a elevação do membro inferior faz um ângulo de $35^{\circ}$ a $70^{\circ}$ com o plano horizontal. A sua positividade a $60^{\circ}$ comprova compressão radicular.

Teste de Romberg: é pedido ao doente para permanecer de pé com os pés juntos, mãos ao lado do corpo e olhos fechados por um minuto. O teste é considerado positivo quando se observa o doente balançar ou mesmo cair.
} 


\section{RESULTADOS}

Dos 135 enfermeiros, $86.7 \%$ pertenciam ao género feminino e a média de idades rondava os 34 anos $(\mathrm{DP}=7.97)$. Apresentavam valores médios de peso e altura (64 $\mathrm{kg}$ e $164 \mathrm{~cm}$, respetivamente) e em termos de categoria profissional, a maioria são enfermeiros (51.9\%) e enfermeiros graduados $(40 \%)$, trabalham nos serviços de cirurgia geral, medicina interna e urgência. $\mathrm{O}$ tempo médio de exercício da profissão era de dez anos, a modalidade de trabalho é por turnos $(80.7 \%)$ e $45.2 \%$ possui segundo emprego. A carga horária média encontrada era de cerca de 48 horas semanais $(\mathrm{DP}=10.6)$, e cerca de $59 \%$ dos enfermeiros admite ter duas pausas ao longo do seu turno de trabalho ao invés de $29.6 \%$ que refere apenas uma.

No que concerne à prevalência da dor lombar nos últimos 12 meses, constatámos que estava presente em $60.7 \%(\mathrm{n}=82)$ dos enfermeiros, contudo nos últimos sete dias apenas $37.8 \%$ refere essa sintomatologia. A análise da gravidade da dor, mostra que $57 \%$ destes profissionais refere sentir dor moderada e $20.7 \%$ diz mesmo ser intensa. Não obstante estas constatações, apenas 5 enfermeiros $(3.7 \%)$ se viram impedidos de realizar o seu trabalho profissional, nos últimos 12 meses, pelas razões apontadas.

\section{Gráfico 1}

Dor lombar (últimos 12 meses e 7 dias) e absentismo (últimos 12 meses)

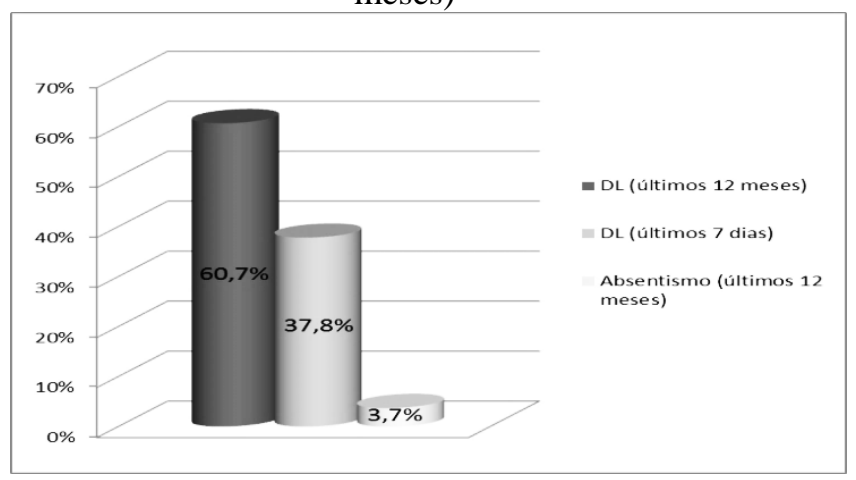

Verificamos ainda que as atividades de enfermagem mais praticadas pelos enfermeiros (mais de cinco vezes por dia) são a administração de medicação (55\%), avaliação de sinais vitais $(54.5 \%)$ e posicionamento/mobilização dos doentes na cama (61.5\%). Por sua vez, 
aquelas que estes consideram mais difíceis de realizar são o posicionamento/mobilização do doente na cama (30.4\%) e o levante do doente sem qualquer ajuda mecânica $(37.8 \%)$.

As atividades de enfermagem, mais relacionadas com a ocorrência das lombalgias são: o inclinar o tronco (86.6\%), o levantar e deslocar cargas superiores a $20 \mathrm{Kg}(85.4 \%)$, rodar o tronco $(76.8 \%)$, o levantar e deslocar cargas entre 10 a $20 \mathrm{Kg}(75.6 \%)$, o manipular cargas superiores a $4 \mathrm{Kg}$ $(69.5 \%)$ e o trabalho de pé (65.9\%).

Os dados apurados no momento da avaliação clínica, mostram que $89.6 \%$ dos participantes referia lombalgia; destes, $30.2 \%$ têm episódios uma ou mais vezes por mês e menos que uma vez por semana, $32.6 \%$ têm episódios com duração inferior a 24 horas, $90.5 \%$ refere ter dor moderada e $41.9 \%$ dor tipo moinha. De referir que nesta avaliação clinica foram encontrados quatro enfermeiros com radiculopatia, seis com teste de Valsalva positivo e um com testes de Laségue e de Romberg positivos. O principal fator atenuante da lombalgia para $51.2 \%$ dos enfermeiros é o repouso, seguindo-se (para 44.3\%) as medidas farmacológicas e não farmacológicas e contrariamente a exacerbação dos sintomas é na opinião de $62.8 \%$ destes profissionais o esforço físico exigido.

As associações significativas encontradas entre as lombalgias e as variáveis independentes, relacionam-se fundamentalmente com o tipo de horário, grupos etários, tempo de profissão e altura. Assim, os resultados revelam que: há um aumento da ocorrência de lombalgia associada ao trabalho por turnos $(\mathrm{p}=0.044)$; que existe uma maior propensão para lombalgia nos enfermeiros com idade inferior a 40 anos $(\mathrm{p}=0.016)$ e com menos tempo de profissão ( $\mathrm{p}=0.005)$; os enfermeiros que afirmam sofrer de lombalgia são em média mais altos do que os que não sofrem $(\mathrm{p}=0.035)$.

Também entre a frequência das atividades de enfermagem desenvolvidas ao longo do dia e a ocorrência de lombalgia, se encontraram duas associações estatisticamente significativas: são elas o posicionamento/mobilização do doente na cama $(\mathrm{p}=0.026)$ e o levantar o doente da cama sem ajuda mecânica $(\mathrm{p}=0.004)$.

Inversamente, não foram encontradas significâncias estatísticas para o tipo de serviço, categoria profissional, carga horária semanal, número de pausas por turno, possuir segundo emprego, género e peso corporal. Do mesmo modo, não foram encontradas associações entre os fatores de risco em saúde (atividade física, fumar, consumo de bebidas alcoólicas e café) e a prevalência de dor lombar. 


\section{DISCUSSÃO}

A reflexão crítica dos resultados deste estudo confirma a existência de uma elevada prevalência de lombalgias de esforço nos enfermeiros que trabalham em contexto hospitalar, em Portugal.

Atividades como posicionar/mobilizar o doente na cama e levantar o doente da cama sem ajuda mecânica, que se revelam estatisticamente influentes na presença de lombalgia nos enfermeiros, são também aquelas que os enfermeiros consideram mais difíceis de executar. Tarefas que impliquem inclinar ou rodar o tronco, deslocar ou manipular cargas pesadas e manter-se de pé, são os fatores que na opinião dos Enfermeiros mais contribuem para o aparecimento das lombalgias.

Estes achados mostram a influência do esforço físico e das posturas corporais inadequadas na presença de dor lombar. Consequentemente, remetem-nos para a elevada importância que as caraterísticas reais do trabalho de enfermagem assumem nesta problemática, devendo assim destacar-se das outras variáveis na abordagem preventiva/interventiva deste fenómeno.

Os dados da avaliação clínica são concordantes e reforçam aqueles que foram obtidos através do autopreenchimento do questionário, sobretudo no que respeita à caraterização da dor. Porém, através do cruzamento de ambos, é possível verificar que 36 dos 48 enfermeiros submetidos à segunda fase de avaliação, mantém dor lombar.

Em síntese podemos afirmar, que estamos perante um grupo de enfermeiros que têm frequentemente lombalgia não específica, de ritmo mecânico, com episódios de curta duração, com dor de intensidade moderada a intensa, com manutenção deste quadro por períodos superiores a um ano.

Os dados no seu conjunto, para além de reforçarem a ideia de que os fatores profissionais, nomeadamente os ligados às condições de trabalho, como a aplicação de força, o tipo de tarefas e suas caraterísticas, levantam a hipótese de muitos destes enfermeiros terem já lombalgia crónica. Este facto deveria, em nossa opinião, ser validado com maior rigor através de estudos do tipo longitudinal, e estendidos a outras realidades profissionais e a novas amostras. Contudo, não deixa de ser um indício relevante, que quando associado ao absentismo inexpressivo (cinco casos) pode significar que os enfermeiros trabalham com dor lombar e continuam a sua atividade profissional, apesar da dor mantida, encarando-a como "normal" para a sua profissão. Esta questão é debatida por alguns autores ao referir que o trabalho não é imutável e as doenças profissionais ou 
relacionadas com o trabalho não são uma inevitabilidade. Pelo contrário, estes autores defendem que é importante o conhecimento aprofundado sobre as caraterísticas do trabalho e dos profissionais, de modo a adaptar as condições deste ao ser humano, garantindo que a sua atividade seja menos prejudicial para a sua saúde, não comprometendo a qualidade da prestação de cuidados aos doentes e a sua segurança (Serranheira, Cotrim, et al., 2012).

\section{CONSIDERAÇÕES FINAIS}

As lombalgias constituem uma entidade nosológica, com grande impacto na profissão de enfermagem, causando dor, limitação funcional e custos elevados com os cuidados de saúde. Os enfermeiros em contexto hospitalar desenvolvem trabalho envolvendo esforços excessivos e repetitivos, durante longos períodos de tempo, adotando por vezes algumas posturas incorretas. A escassez de meios técnicos e humanos, aliados às restritas condições de trabalho e aos fatores de risco individuais e socioculturais, constituem-se como relevantes fatores de risco das lombalgias.

Assim é premente a necessidade de olhar para as particularidades da atividade profissional de enfermagem, em especial no contexto hospitalar, no sentido de prevenir a lombalgia nestes profissionais e a incapacidade que lhe pode estar associada. Deve ainda ter-se em conta as caraterísticas reais do contexto de trabalho, mas também a responsabilidade que cabe aos próprios profissionais em si, no desenvolvimento das suas intervenções, no sentido de minimizar o impacto negativo do trabalho na saúde destes e prevenir a incidência de novos casos de dor lombar.

\section{BIBLIOGRAFIA}

Alexopoulos, E. C., Burdorf, A., \& Kalokerinou, A. (2003). Risk factors for musculoskeletal disorders among nursing personnel in Greek hospitals. Int Arch Occup Environ Health, 76(4), 289-294. doi: http://dx.doi.org/10.1007/s00420-003-0442-9

Alexopoulos, E. C., Burdorf, A., \& Kalokerinou, A. (2006). A comparative analysis on musculoskeletal disorders between Greek and Dutch nursing personnel. Int Arch Occup Environ Health, 79(1), 8288. doi: http://dx.doi.org/10.1007/s00420-005-0033-z 
Bos, E., Krol, B., van der Star, L., \& Groothoff, J. (2007). Risk factors and musculoskeletal complaints in non-specialized nurses, IC nurses, operation room nurses, and X-ray technologists. Int Arch Occup Environ Health, 80(3), 198-206. doi: http://dx.doi.org/10.1007/s00420-006-0121-8

Branney, J., \& Newell, D. (2009). Back pain and associated healthcare seeking behaviour in nurses: A survey. Clinical Chiropractic, 12(4), 130-143. doi: http://dx.doi.org/10.1016/j.clch.2009.12.002

Dagenais, S., Caro, J., \& Haldeman, S. (2008). A systematic review of low back pain cost of illness studies in the United States and $\begin{array}{lllll}\text { internationally. Spine } J, & 8(1), & 8-20 . & \text { doi: }\end{array}$ http://dx.doi.org/10.1016/j.spinee.2007.10.005

Daraiseh, N. M., Cronin, S. N., Davis, L. S., Shell, R. L., \& Karwowski, W. (2010). Low back symptoms among hospital nurses, associations to individual factors and pain in multiple body regions. International Journal of Industrial Ergonomics, 40(1), 19-24. doi: http://dx.doi.org/10.1016/j.ergon.2009.11.004

Fonseca, R., \& Serranheira, F. (2006). Sintomatologia musculoesquelética auto-referida por enfermeiros em meio hospitalar. Revista Portuguesa de Saúde Pública, 6, 37-44.

Hoy, D., Brooks, P., Blyth, F., \& Buchbinder, R. (2010). The Epidemiology of low back pain. Best Practice \& Research Clinical Rheumatology, 24(6), 769-781. doi: http://dx.doi.org/10.1016/j.berh.2010.10.002

Karahan, A., \& Bayraktar, N. (2004). Determination of the usage of body mechanics in clinical settings and the occurrence of low back pain in nurses. International Journal of Nursing Studies, 41(1), 67-75. doi: http://dx.doi.org/10.1016/S0020-7489(03)00083-X

Lorusso, A., Bruno, S., \& L'Abbate, N. (2007). A review of low back pain and musculoskeletal disorders among Italian nursing personnel. Ind Health, 45(5), 637-644.

Magnago, T. S. B. d. S., Lisboa, M. T. L., Griep, R. H., Kirchhof, A. L. C., Camponogara, S., Nonnenmacher, C. d. Q., \& Vieira, L. B. (2010). Condições de trabalho, características sociodemográficas e distúrbios musculoesqueléticos em trabalhadores de enfermagem. Acta Paulista de Enfermagem, 23, 187-193.

Maul, I., Laubli, T., Klipstein, A., \& Krueger, H. (2003). Course of low back pain among nurses: a longitudinal study across eight years. Occup Environ Med, 60(7), 497-503. 
Ratzon, N. Z., \& Froom, P. (2006). Postural control in nurses with and without low back pain. Work, 26(2), 141-145.

Serranheira, F., Cotrim, T., Rodrigues, V., Nunes, C., \& Sousa-Uva, A. (2012). Lesões musculoesqueléticas ligadas ao trabalho em enfermeiros portugueses: «ossos do ofício» ou doenças relacionadas com o trabalho? Revista Portuguesa de Saúde Pública, 30(2), 193203. doi: http://dx.doi.org/10.1016/j.rpsp.2012.10.001

Serranheira, F., Sousa-Uva, M., \& Sousa-Uva, A. (2012). Lombalgias e trabalho hospitalar em enfermeiro (a) s. Revista Brasileira de Medicina do Trabalho, 10(2), 80-87.

Sikiru, L., \& Shmaila, H. (2009). Prevalence and risk factors of low back pain among nurses in Africa: Nigerian and Ethiopian specialized hospitals survey study. East Afr J Public Health, 6(1), 22-25.

Smedley, J., Egger, P., Cooper, C., \& Coggon, D. (1995). Manual handling activities and risk of low back pain in nurses. Occup Environ Med, 52(3), 160-163.

Tinubu, B. M., Mbada, C. E., Oyeyemi, A. L., \& Fabunmi, A. A. (2010). Work-related musculoskeletal disorders among nurses in Ibadan, South-west Nigeria: a cross-sectional survey. BMC Musculoskelet Disord, 11, 12. doi: http://dx.doi.org/10.1186/1471-2474-11-12 